\title{
Wide-azimuth angle gathers for wave-equation migration ${ }^{a}$
}

${ }^{a}$ Published in Geophysics, 76, no. 3, S131-S141, (2011)

Paul Sava (Center for Wave Phenomena, Colorado School of Mines) Ioan Vlad (currently Fusion Petroleum Technologies, formerly Statoil)

\begin{abstract}
Extended common-image-point-gathers (CIP) contain all the necessary information for decomposition of reflectivity as a function of the reflection and azimuth angles at selected locations in the subsurface. This decomposition operates after the imaging condition applied to wavefields reconstructed by any type of wideazimuth migration method, e.g. using downward continuation or time reversal. The reflection and azimuth angles are derived from the extended images using analytic relations between the space-lag and time-lag extensions. The transformation amounts to a linear Radon transform applied to the CIPs obtained after the application of the extended imaging condition. If information about the reflector dip is available at the CIP locations, then only two components of the space-lag vectors are required, thus reducing computational cost and increasing the affordability of the method. Applications of this method include the study of subsurface illumination in areas of complex geology where ray-based methods are not usable, and the study of amplitude variation with reflection and azimuth angles if the subsurface subsurface illumination is sufficiently dense. Migration velocity analysis could also be implemented in the angle domain, although an equivalent implementation in the extended domain is cheaper and more effective.
\end{abstract}

\section{INTRODUCTION}

In regions characterized by complex subsurface structure, wave-equation depth migration is a powerful tool for accurately imaging the earth's interior. The quality of the final image greatly depends on the quality of the velocity model and on the quality of the technique used for wavefield reconstruction in the subsurface (Gray et al., 2001).

However, structural imaging is not the only objective of wave-equation imaging. It is often desirable to construct images depicting reflectivity as a function of reflection angles. Such images not only highlight the subsurface illumination patterns, but could potentially be used for image postprocessing for amplitude variation with angle 
analysis. Furthermore, angle domain images can be used for tomographic velocity updates.

Angle gathers can be produced either using ray methods (Xu et al., 1998; BrandsbergDahl et al., 2003) or by using wavefield methods (de Bruin et al., 1990; Mosher et al., 1997; Prucha et al., 1999; Xie and Wu, 2002; Rickett and Sava, 2002; Sava and Fomel, 2003; Biondi and Symes, 2004; Wu and Chen, 2006). Gathers constructed with these methods have similar characteristics since they simply describe the reflectivity as a function of incidence angles at the reflector. However, as indicated by Stolk and Symes (2004), even in perfectly known but strongly refracting media angle gathers are damaged by undersampling of data on the surface, regardless of the method used for their construction. In this paper, we address the problem of wavefield-based angle decomposition.

Angle decomposition can be applied either before or after the application of an imaging condition. The two classes of methods differ by the objects used to study the angle-dependent illumination of subsurface geology. The methods operating before the imaging condition decompose the extrapolated wavefields from the source and receivers (de Bruin et al., 1990; Mosher et al., 1997; Prucha et al., 1999; Wu and Chen, 2006). This type of decomposition is costly since it operates on individual wavefields characterized by complex multipathing. In contrast, the methods operating after the imaging condition decompose the images themselves which are represented as a function of space and additional parameters, typically refered to as extensions (Rickett and Sava, 2002; Sava and Fomel, 2003, 2006, Sava and Vasconcelos, 2011). In the end, the various classes of methods lead to similar representations of the angle-dependent reflectivity represented by the so-called scattering matrix. The main differences lie in the complexity of the decomposition and in the cost required to achieve this result. In this paper, we focus on angle decomposition of extended images.

Conventionally, angle-domain imaging uses common-image-gathers (CIGs) describing the reflectivity as a function of reflection angles and a space axis, typically the depth axis. An alternative way of constructing angle-dependent reflectivity is based on common-image-point-gathers (CIP) selected at various positions in the subsurface. As pointed out by Sava and Vasconcelos (2011), CIPs are advantageous because they sample the image at the most relevant locations (along the main reflectors), they avoid computations at locations that are not useful for further analysis (inside salt bodies), they can have higher density at locations where the structure is more complex and lower density in areas of poor illumination, and they avoid the depth bias typical for gathers constructed as a function of the depth axis. In this paper, we focus on angle decomposition using extended CIPs.

A recent development in wave-equation imaging is the use of wide-azimuth data (Regone, 2006; Michell et al., 2006; Clarke et al., 2006). Imaging with such data poses additional challenges for angle-domain imaging, mainly arising from the larger data size and the interpretation difficulty of data of higher dimensionality. Several techniques have been proposed for wide-azimuth angle decomposition, including ray-based methods (Koren et al. 2008) and wavefield methods using wavefield decomposition 
before imaging (Zhu and Wu, 2010; Biondi and Tisserant, 2004) or after imaging (Sava and Fomel, 2005). Here, we complete the set of techniques available for angle gather construction by describing an algorithm applicable to extended common-image-pointgathers.

\section{IMAGING CONDITIONS}

Conventional seismic imaging is based on the concept of single scattering. Under this assumption, waves propagate from seismic sources, interact with discontinuities and return to the surface as reflected seismic waves. We commonly speak about a "source" wavefield, originating at the seismic source and propagating in the medium prior to any interaction with discontinuities, and a "receiver" wavefield, originating at discontinuities and propagating in the medium to the receivers (Berkhout, 1982; Clærbout, 1985). The two wavefields kinematically coincide at discontinuities.

We can formulate imaging as a process involving two steps: the wavefield reconstruction and the imaging condition. The key elements in this imaging procedure are the source and receiver wavefields, $W_{s}$ and $W_{r}$ which are 4-dimensional objects as a function of space $\mathbf{x}=\{x, y, z\}$ and time $t$, or as a function of space and frequency $\omega$. For imaging, we need to analyze if the wavefields match kinematically in time and then extract the reflectivity information using an imaging condition operating along the space and time axes.

A conventional cross-correlation imaging condition (cIC) based on the reconstructed wavefields can be formulated in the time or frequency domain as the zero lag of the cross-correlation between the source and receiver wavefields (Clærbout, 1985):

$$
\begin{aligned}
R(\mathbf{x}) & =\sum_{\text {shots }} \sum_{t} W_{s}(\mathbf{x}, t) W_{r}(\mathbf{x}, t) \\
& =\sum_{\text {shots }} \sum_{\omega} \overline{W_{s}(\mathbf{x}, \omega)} W_{r}(\mathbf{x}, \omega),
\end{aligned}
$$

where $R$ represents the migrated image and the over-line represents complex conjugation. This operation exploits the fact that portions of the source and receiver wavefields match kinematically at subsurface positions where discontinuities occur. Alternative imaging conditions use deconvolution of the source and receiver wavefields, but we do not elaborate further on this subject since the differences between cross-correlation and deconvolution are not central for this paper.

An extended imaging condition preserves in the output image certain acquisition (e.g. source or receiver coordinates) or illumination (e.g. reflection angle) parameters (Clayton and Stolt, 1981, Clærbout, 1985, Stolt and Weglein, 1985, Weglein and Stolt, 1999). In shot-record migration, the source and receiver wavefields are reconstructed on the same computational grid at all locations in space and all times or frequencies, therefore there is no a-priori wavefield separation that can be transferred to the output image. In this situation, the separation can be constructed by correlation of the 
wavefields from symmetric locations relative to the image point, measured either in space (Rickett and Sava, 2002; Sava and Fomel, 2005) or in time (Sava and Fomel, 2006). This separation essentially represents local cross-correlation lags between the source and receiver wavefields. Thus, an extended cross-correlation imaging condition (eIC) defines the image as a function of space and cross-correlation lags in space and time. This imaging condition can also be formulated in the time and frequency domains (Sava and Vasconcelos, 2011):

$$
\begin{aligned}
R(\mathbf{x}, \boldsymbol{\lambda}, \tau) & =\sum_{\text {shots }} \sum_{t} W_{s}(\mathbf{x}-\boldsymbol{\lambda}, t-\tau) W_{r}(\mathbf{x}+\boldsymbol{\lambda}, t+\tau) \\
& =\sum_{\text {shots }} \sum_{\omega} e^{2 i \omega \tau} \overline{W_{s}(\mathbf{x}-\boldsymbol{\lambda}, \omega)} W_{r}(\mathbf{x}+\boldsymbol{\lambda}, \omega) .
\end{aligned}
$$

Equations 1 2 2 represent a special case of equations 3 , 4 for $\boldsymbol{\lambda}=0$ and $\tau=0$. Assuming that all errors accumulated in the incorrectly-reconstructed wavefields are due to the velocity model, the extended images could be used for velocity model building by exploiting semblance properties emphasized by the space-lags (Biondi and Sava, 1999; Shen et al., 2003; Sava and Biondi, 2004a b) and focusing properties emphasized by the time-lag (Faye and Jeannot, 1986; MacKay and Abma, 1992, 1993; Nemeth, 1995, 1996). Furthermore, these extensions can be converted to reflection angles (Weglein and Stolt, 1999; Sava and Fomel, 2003, 2006), thus enabling analysis of amplitude variation with angle for images constructed in complex areas using wavefield-based imaging.

Typically, angle decomposition with extended images uses common image gathers, i.e. representations of (a subset of) the extensions as a function of a space axis, typically the depth axis. As pointed out by Sava and Vasconcelos (2011), this approach suffers from major drawbacks. Common-image-gathers are appropriate for nearly horizontal structures and they are computationally wasteful since they require un-necessary calculations, e.g. inside massive salt bodies. In contrast, the commonimage-point-gathers advocated by Sava and Vasconcelos (2011) are constructed at selected points in the image, thus eliminating unnecessary calculations, and they can accomodate arbitrary orientations of the reflectors.

In this paper, we use extended common-image-point-gathers to extract angledependent reflectivity at individual points in the image. The method described in the following section is appropriate for $3 \mathrm{D}$ wide-azimuth wave-equation imaging. The problem we are solving is to decompose extended CIPs as a function of azimuth $\phi$ and reflection $\theta$ angles at selected points in the image. In general, the input for such decomposition are gathers in the $\{\boldsymbol{\lambda}, \tau\}$ domain, and the output are gathers in the $\{\phi, \theta, \tau\}$ domain:

$$
R(\boldsymbol{\lambda}, \tau) \Longrightarrow R(\phi, \theta, \tau) \text {. }
$$

Here we address a special case of this decomposition which is appropriate for imaging with correct velocity. In this case, all the energy in the output CIPs concentrates at $\tau=0$, so we can focus our attention on a particular case of the decomposition 
which does not preserve the time-lag variable in the output: $R(\boldsymbol{\lambda}, \tau) \Longrightarrow R(\phi, \theta)$. We omit the dependence of the extended images on space $(\mathbf{x})$ to highlight the fact that the decomposition can be performed independently at various points in the image. The topic of angle decomposition when the gathers are constructed with incorrect velocity remains outside the scope of this paper. However, we note that the angle decomposition in such situation is not necessary, since semblance optimization can be implemented based on the image extensions directly (Shen and Symes, 2008; Symes, 2009).

In the remainder of the paper, we show that all the necessary information for this decomposition is available after wave-equation migration, regardless of its implementation, e.g by depth or time extrapolation. A pre-requisite for this decomposition is the moveout function characterizing individual shots, as discussed in the following section. We then show how the moveout information can be used for angle decomposition and illustrate the method with simple and complex synthetic examples.

\section{MOVEOUT FUNCTION}

In this section, we derive the formula for the moveout function characterizing reflections in the extended $\{\boldsymbol{\lambda}, \tau\}$ domain. The purpose of this derivation is to find a procedure for angle decomposition, i.e. a representation of reflectivity as a function of reflection and azimuth angles.

An implicit assumption made by all methods of angle decomposition is that we can describe the reflection process by locally planar objects. Such methods assume that (locally) the reflector is a plane, and that the incident and reflected wavefields are also (locally) planar. Only with these assumptions we can define vectors in-between which we measure angles like the angles of incidence and reflection, as well as the azimuth angle of the reflection plane. Our method uses this assumption explicitly. However, we do not assume that the wavefronts are planar. Instead, we consider each (complex) wavefront as a superposition of planes with different orientations. In the following, we discuss how each one of these planes would behave during the extended imaging and angle decomposition. Thus, our method applies equally well for simple and complex wavefields characterized by multipathing.

We define the following unit vectors to describe the reflection geometry and the conventional and extended imaging conditions:

- $\mathbf{n}$ : a unit vector aligned with the reflector normal;

- a: a unit vector representing the projection of the azimuth vector $\mathbf{v}$ in the reflector plane;

- $\mathbf{n}_{s}$ : a unit vector orthogonal to the source wavefront;

- $\mathbf{n}_{r}$ : a unit vector orthogonal to the receiver wavefront; 
- q: a unit vector at the intersection of the reflection plane and the reflector plane.

By construction, vectors $\mathbf{n}, \mathbf{n}_{s}, \mathbf{n}_{r}$ and $\mathbf{q}$ are co-planar and vectors $\mathbf{n}$ and $\mathbf{q}$ are orthogonal, Figure 1.

With these definitions, the (planar) source and receiver wavefields are given by the expressions:

$$
\begin{aligned}
& \mathbf{n}_{s} \cdot \mathbf{x}=v t_{s} \\
& \mathbf{n}_{r} \cdot \mathbf{x}=v t_{r} .
\end{aligned}
$$

Here, $\mathbf{x}$ are space coordinates, $t_{s}$ and $t_{r}$ are times defining the planes under consideration, and $v$ represents velocity. Equations 6 and 7 define the conventional imaging condition given by equations 1 and 2. This condition states that an image is formed when the source and receiver wavefields are time-coincident at reflection points. In Equations 6 and 7, we explicitly impose the condition that the source and receiver planes and the reflector plane intersect at the image point.

Similarly, we can rewrite the extended imaging condition using the planar approximation of the source and receiver wavefields using the expressions:

$$
\begin{aligned}
& \mathbf{n}_{s} \cdot(\mathbf{x}-\boldsymbol{\lambda})=v\left(t_{s}-\tau\right) \\
& \mathbf{n}_{r} \cdot(\mathbf{x}+\boldsymbol{\lambda})=v\left(t_{r}+\tau\right)
\end{aligned}
$$

As discussed earlier, $\boldsymbol{\lambda}$ and $\tau$ are space- and time-lags, and $v$ represents the local velocity at the image point, assumed to be constant in the immediate vicinity of this point. This assumption is justified by the need to operate with planar objects, as indicated earlier. With this construction, the source and receiver planes are shifted relative to one-another by equal quantities in the positive and negative directions and in space and time, equations 3.4 .

We can eliminate the space variable $\mathbf{x}$ by substituting equation 6 in equation 8 and equation 7 in equation 9 .

$$
\begin{aligned}
& \mathbf{n}_{s} \cdot \boldsymbol{\lambda}=v \tau \\
& \mathbf{n}_{r} \cdot \boldsymbol{\lambda}=v \tau
\end{aligned}
$$

Furthermore, we can re-arrange the system given by equations 10 and 11 by sum and difference of the equations:

$$
\begin{aligned}
& \left(\mathbf{n}_{s}+\mathbf{n}_{r}\right) \cdot \boldsymbol{\lambda}=2 v \tau \\
& \left(\mathbf{n}_{s}-\mathbf{n}_{r}\right) \cdot \boldsymbol{\lambda}=0
\end{aligned}
$$

So far, we have not assumed any relation between the vectors characterizing the source and receiver planes, $\mathbf{n}_{s}$ and $\mathbf{n}_{r}$. However, if the source and receiver wavefields 
correspond to a reflection from a planar interface, these vectors are not independent of one-another, but are related by Snell's law which can be formulated as

$$
\mathbf{n}_{r}=\mathbf{n}_{s}-2\left(\mathbf{n}_{s} \cdot \mathbf{n}\right) \mathbf{n}
$$

This relations follows from geometrical considerations and it is based on the conservation of ray vector projection along the reflector. Equation 14 is only valid for PP reflections in an isotropic medium.

Substituting Snell's law into the system $12, \sqrt{13}$, and after trivial manipulations of the equations, we obtain the system:

$$
\begin{aligned}
{\left[\mathbf{n}_{s}-\left(\mathbf{n}_{s} \cdot \mathbf{n}\right) \mathbf{n}\right] \cdot \boldsymbol{\lambda} } & =v \tau \\
\left(\mathbf{n}_{s} \cdot \mathbf{n}\right)(\mathbf{n} \cdot \boldsymbol{\lambda}) & =0
\end{aligned}
$$

In general, the plane characterizing the source wavefield is not orthogonal to the reflection plane (there would be no reflection in that case), therefore we can simplify equation 16 by dropping the term $\left(\mathbf{n}_{s} \cdot \mathbf{n}\right) \neq 0$. Moreover, we can replace in equation 15 the expression in the square bracket with the quantity $\mathbf{q} \sin \theta$, where $\mathbf{q}$ is the unit vector characterizing the line at the intersection of the reflection and reflector planes, and $\theta$ is the reflection angle contained in the reflection plane. With these simplifications, the system 15,16 can be re-written as:

$$
\begin{aligned}
(\mathbf{q} \cdot \boldsymbol{\lambda}) \sin \theta & =v \tau, \\
\mathbf{n} \cdot \boldsymbol{\lambda} & =0 .
\end{aligned}
$$

The system 17 - 18 allows for a straightforward physical interpretation of the extended imaging condition. First, the expression 18 indicates that of all possible space-lags that can be applied to the reconstructed wavefields, the only ones that contribute to the extended image are those for which the space-lag vector $\boldsymbol{\lambda}$ is orthogonal to the reflector normal vector $\mathbf{n}$. Furthermore, assuming that the space-shift applied to the source and receiver planes is contained in the reflector plane, i.e. $\boldsymbol{\lambda} \perp \mathbf{n}$, then the expression 17 describe the moveout function in an extended gather as a function of the space-lag $\boldsymbol{\lambda}$, the time lag $\tau$, the reflection angle $\theta$, the orientation vector $\mathbf{q}$. The vector $\mathbf{q}$ is orthogonal to the reflector normal and depends on the reflection azimuth angle $\phi$.

Figures 1 , 3 illustrate the process involved in the extended imaging condition and describe pictorially its physical meaning. Figure 1 shows the source and receiver planes, as well as the reflector plane together with their unit vector normals. Figure 2 shows the source and receiver planes displaced by the space lag vector $\boldsymbol{\lambda}$ contained in the reflector plane, as indicated by equation 18 . The displaced planes do not intersect at the reflection plane, thus they do not contribute to the extended image at this point. However, with the application of time shifts with the quantity $\tau=(\mathbf{q} \cdot \boldsymbol{\lambda}) \sin \theta / v$, i.e. a translation in the direction of plane normals, the source and receiver planes are restored to the image point, thus contributing to the extended image, Figure 3. 


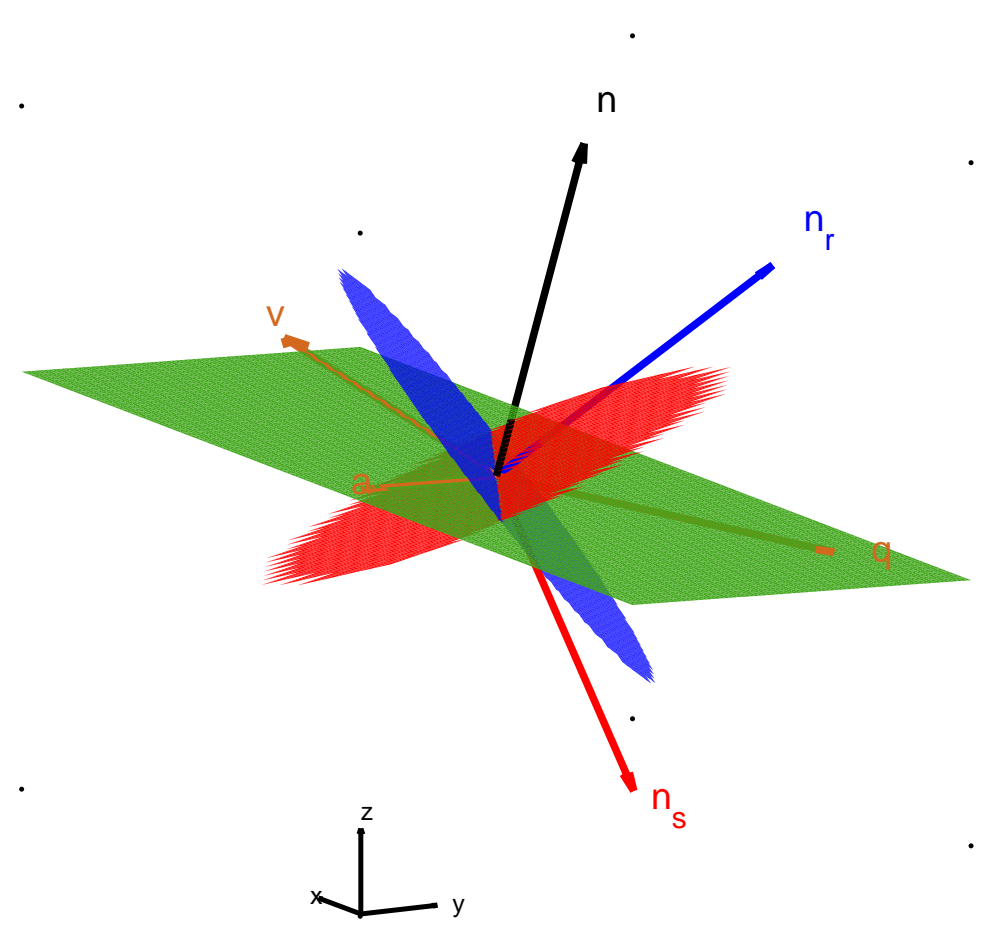

Figure 1: The reflector plane (of normal $\mathbf{n}$ ), together with the source and receiver planes (of normals $\mathbf{n}_{s}$ and $\mathbf{n}_{r}$, respectively). The figure represents the source/receiver planes in their original position, i.e. as obtained by wavefield reconstruction.

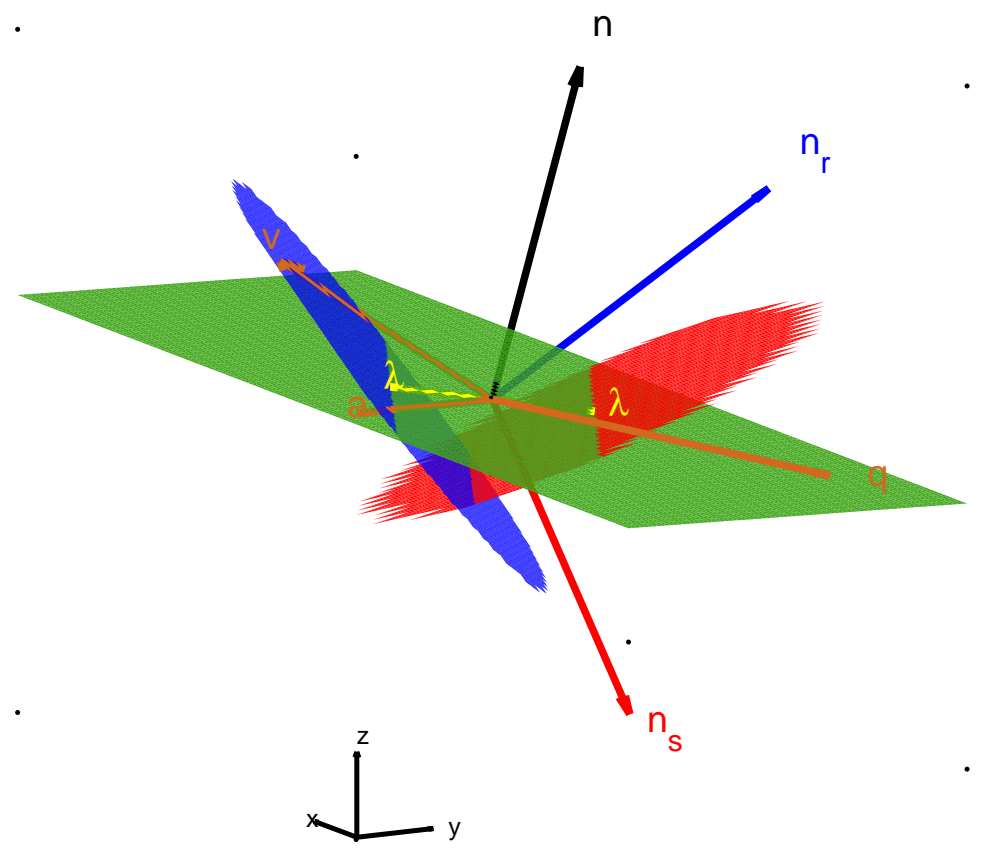

Figure 2: The reflector plane (of normal $\mathbf{n}$ ), together with the source and receiver planes (of normals $\mathbf{n}_{s}$ and $\mathbf{n}_{r}$, respectively). The figure represents the source/receiver planes displaced with the space-lag $\boldsymbol{\lambda}$ constrained in the reflector plane. 


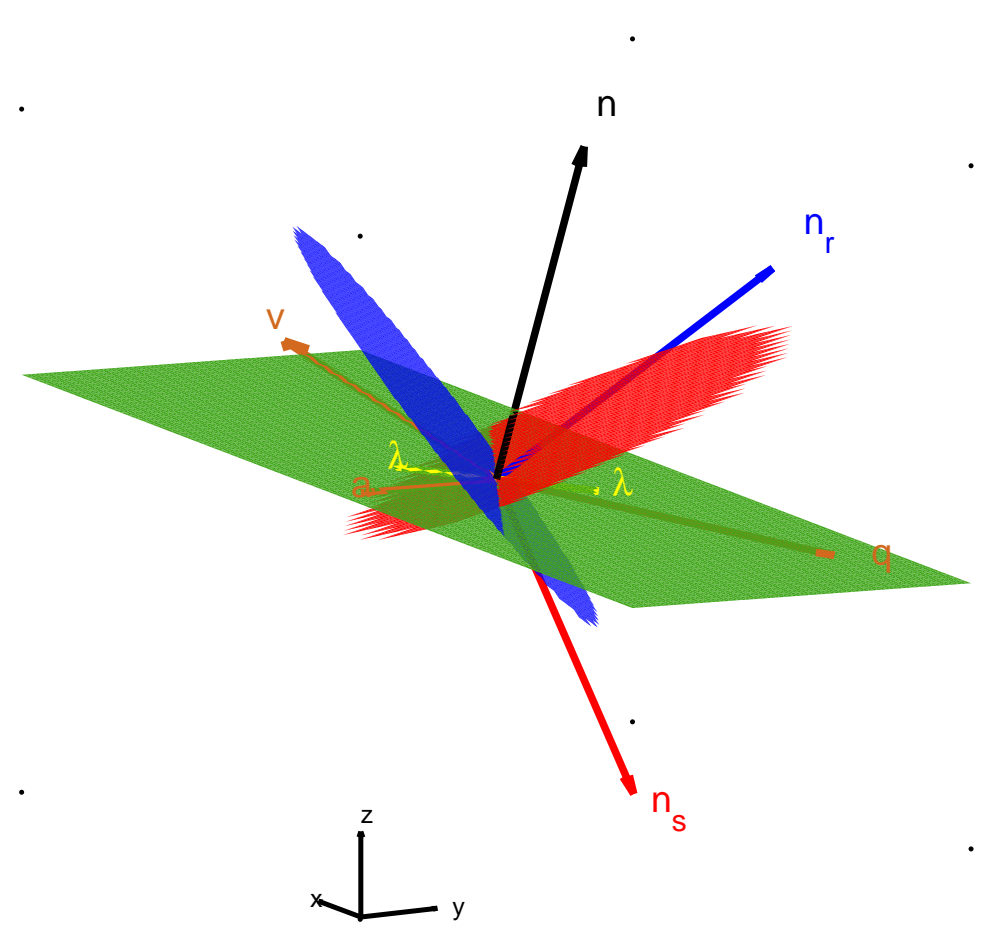

Figure 3: The reflector plane (of normal $\mathbf{n}$ ), together with the source and receiver planes (of normals $\mathbf{n}_{s}$ and $\mathbf{n}_{r}$, respectively). The figure represents the source/receiver planes displaced with space-lag $\boldsymbol{\lambda}$ and time-lag $\tau$. The space and time-lags are related by equation 17 . 


\section{ANGLE DECOMPOSITION}

In this section we discuss the steps required to transform lag-domain CIPs into angledomain CIPs using the moveout function derived in the preceding section. We also present the algorithm used for angle decomposition and illustrate it using a simple $3 \mathrm{D}$ model of a horizontal reflector in a medium with constant velocity which allows us to validate analytically the procedure.

The outer loop of the algorithm is over the CIPs evaluated during migration. The angle decompositions of individual CIPs are independent of one-another, therefore the algorithm is easily parallelizable over the outer loop. At every CIP, we need to access the information about the reflector normal (n) and about the local velocity $(v)$. The reflector dip information can be extracted from the conventional image, and the velocity is the same as the one used for migration.

Prior to the angle decomposition, we also need to define a direction relative to which we measure the reflection azimuth. This direction is arbitrary and depends on the application of the angle decomposition. Typically, the azimuth is defined relative to a reference direction (e.g. North). Here, we define this azimuth direction using an arbitrary vector $\mathbf{v}$. Using the reflector normal (n) we can build the projection of the azimuth vector $(\mathbf{a})$ in the reflector plane as

$$
\mathbf{a}=(\mathbf{n} \times \mathbf{v}) \times \mathbf{n} .
$$

This construction assures that vector $\mathbf{a}$ is contained in the reflector plane (i.e. it is orthogonal on $\mathbf{n}$ ) and that it is co-planar with vectors $\mathbf{n}$ and $\mathbf{v}$, Figure 1. Of course, this construction is just one of the many possible definitions of the azimuth reference. In the following, we measure the azimuth angle $\phi$ relative to vector a and the reflection angle $\theta$ relative to the normal to the reflector given by vector $\mathbf{n}$.

Then, for every azimuth angle $\phi$, using the reflector normal (n) and the azimuth reference (a), we can construct the trial vector $\mathbf{q}$ which lies at the intersection of the reflector and the reflection planes. We scan over all possible vectors $\mathbf{q}$, although only one azimuth corresponds to the reflection from a given shot. This scan ensures that we capture the reflection information from all shots in the survey. Given the reflector normal (the axis of rotation) and the trial azimuth angle $\phi$, we can construct the different vectors $\mathbf{q}$ by the application of the rotation matrix

$Q(\mathbf{n}, \phi)=\left[\begin{array}{ccc}n_{x}^{2}+\left(n_{y}^{2}+n_{z}^{2}\right) \cos \phi & n_{x} n_{y}(1-\cos \phi)-n_{z} \sin \phi & n_{x} n_{z}(1-\cos \phi)+n_{y} \sin \phi \\ n_{y} n_{x}(1-\cos \phi)+n_{z} \sin \phi & n_{y}^{2}+\left(n_{z}^{2}+n_{x}^{2}\right) \cos \phi & n_{y} n_{z}(1-\cos \phi)-n_{x} \sin \phi \\ n_{z} n_{x}(1-\cos \phi)-n_{y} \sin \phi & n_{z} n_{y}(1-\cos \phi)+n_{x} \sin \phi & n_{z}^{2}+\left(n_{x}^{2}+n_{y}^{2}\right) \cos \phi\end{array}\right]$

to the azimuth reference vector a, i.e.

$$
\mathbf{q}=Q(\mathbf{n}, \phi) \mathbf{a}
$$

In this formulation, the normal vector $\mathbf{n}$ of components $\left\{n_{x}, n_{y}, n_{z}\right\}$ can take arbi- 
trary orientations and does not need to be normalized. Then, for every reflection angle $\theta$, we map the lag-domain CIP to the angle-domain by summation over the surface defined by equation 17. This operation represents a planar Radon transform (a slant-stack) over an analytically-defined surface in the $\{\boldsymbol{\lambda}, \tau\}$ space. The output is the representation of the CIP in the angle-domain. In order to preserve the signal bandwidth, the slant-stack needs to use a "rho filter" which compensates the high frequency decay caused by the summation (Clærbout, 1976). The explicit algorithm for angle decomposition is given in Appendix A.

Consider a simple 3D model consisting of a horizontal reflector in a constant velocity medium. We simulate one shot in the center of the model at coordinates $x=4 \mathrm{~km}$ and $y=4 \mathrm{~km}$, with receivers distributed uniformly on the surface on a grid spaced at every $20 \mathrm{~m}$ in the $x$ and $y$ directions. We use time-domain finite-differences for modeling. Figure 4 represents the image obtained by wave-equation migration of the simulated shot using downward continuation. The illumination is limited to a narrow region around the shot due to the limited array aperture.

Figure 4: The image obtained for a horizontal reflector in constant velocity using one shot located in the center of the model.

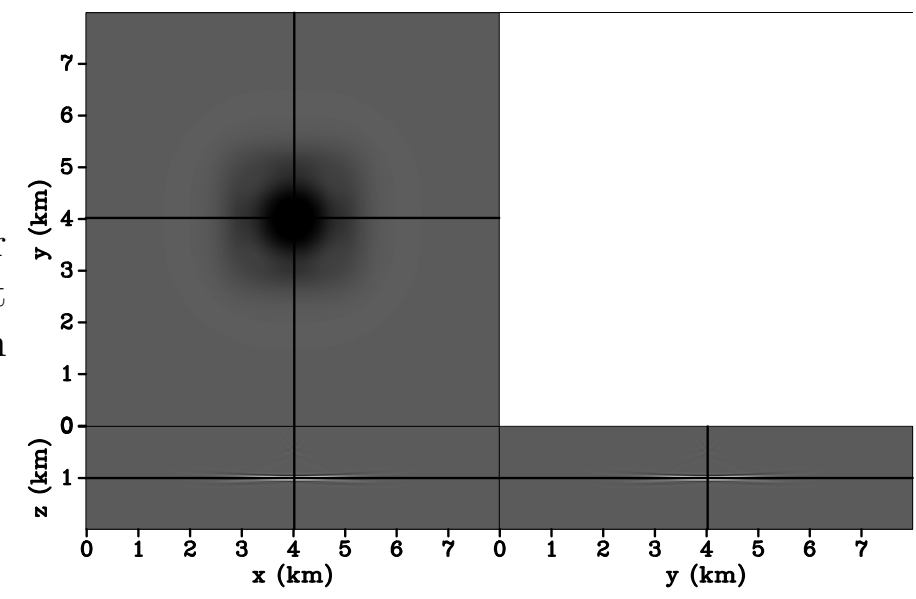

Figures $5 \mathrm{a} 5 \mathrm{5d}$ depict CIPs obtained by migration of the simulated shot at the reflector depth and at coordinates $\{x, y\}$ equal to $\{3.2,3.2\} \mathrm{km},\{3.2,4.8\} \mathrm{km},\{4.8,4.8\} \mathrm{km}$ and $\{4.8,3.2\} \mathrm{km}$, respectively. For these CIPs, the reflection angle is invariant $\theta=48.5^{\circ}$, but the azimuth angles relative to the $x$ axis are $-135^{\circ},+135^{\circ},+45^{\circ}$ and $-45^{\circ}$, respectively. Figures $5 \mathrm{e} 5 \mathrm{~h}$ show the angle decomposition in polar coordinates. Here, we use the trigonometric convention to represent the azimuth angle $\phi$ and we represent the reflection angle in every azimuth in the radial direction (with normal incidence at the center of the plot). Each radial line corresponds to $30^{\circ}$ and each circular contour corresponds to $15^{\circ}$.

Similarly, Figures 6a $6 \mathrm{~d}$ depict CIPs obtained by migration of the simulated shot the reflector depth and at coordinates $\{x, y\}$ equal to $\{2.8,2.8\} \mathrm{km},\{3.2,3.2\} \mathrm{km}$, $\{3.6,3.6\} \mathrm{km}$ and $\{4.0,4.0\} \mathrm{km}$, respectively. For these CIPs, the azimuth angle is invariant $\phi=-135^{\circ}$, but the reflection angles relative to the reflector normal are $59.5^{\circ}, 48.5^{\circ}, 29.5^{\circ}$, and $0^{\circ}$ respectively.

In all examples, the decomposition angles correspond to the theoretical values, 


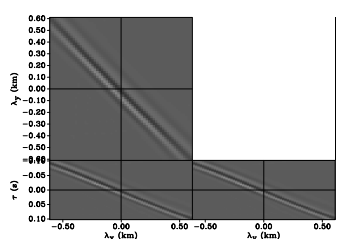

a

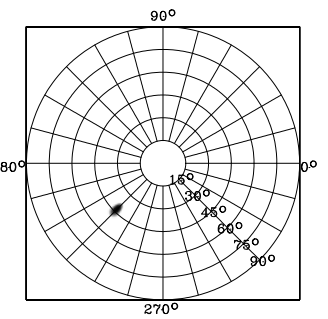

$\mathrm{e}$

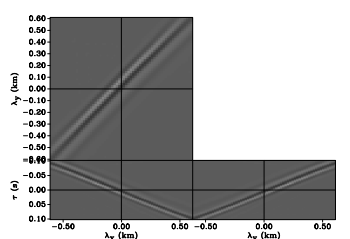

$\mathrm{b}$

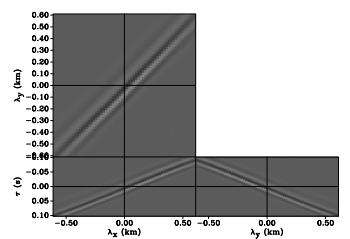

$\mathrm{d}$

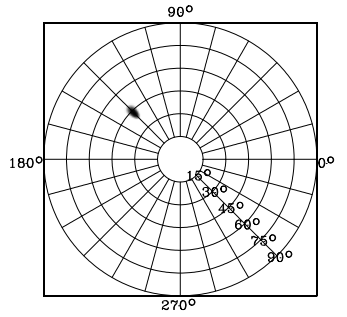

f

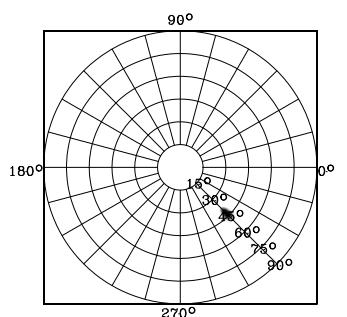

$\mathrm{h}$

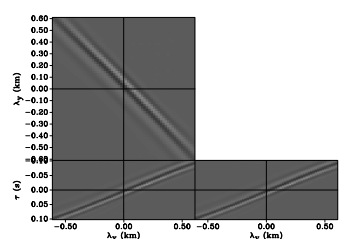

$\mathrm{c}$

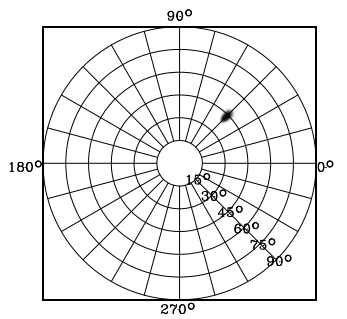

g

Figure 5: Illustration of CIP angle decomposition for illumination at fixed reflection angle. Panels (a)-(d) show lag-domain CIPs, and panels (e)-(f) show angle-domain CIPs in polar coordinates. The angles $\phi$ and $\theta$ are indexed along the contours using the trigonometric convention and along the radial lines increasing from the center. 


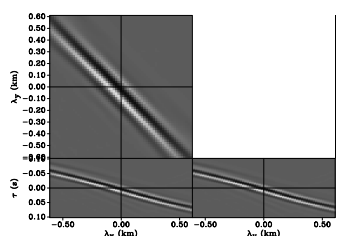

a

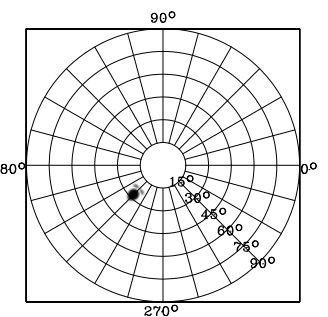

e

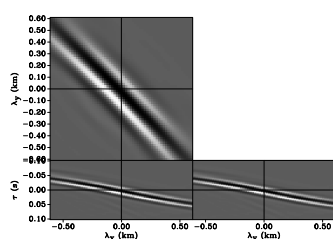

b

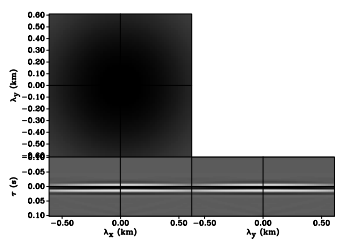

d

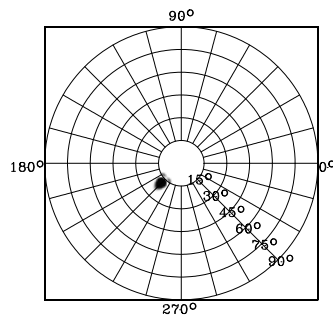

f

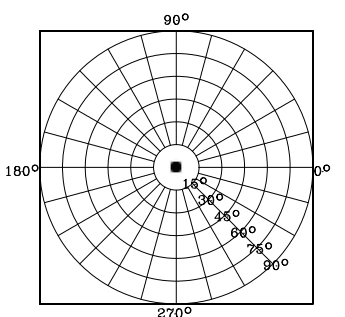

$\mathrm{h}$

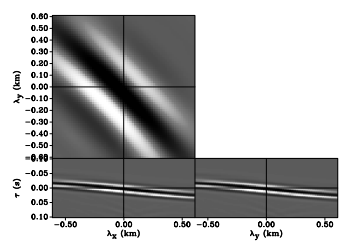

c

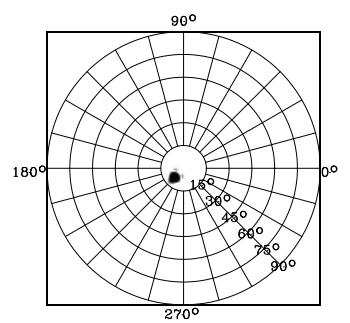

g

Figure 6: Illustration of CIP angle decomposition for illumination at fixed azimuth angle. Panels (a)-(d) show lag-domain CIPs, and panels (e)-(h) show angle-domain CIPs in polar coordinates. The angles $\phi$ and $\theta$ are indexed along the contours using the trigonometric convention and along the radial lines increasing from the center. 
thus confirming the validity of our decomposition.

\section{EXAMPLES}

We illustrate the method discussed in the preceding section with common-imagepoint-gathers constructed using the wide-azimuth SEAM data (). Figure 7 shows the velocity model in the area used for imaging. For demonstration, we consider 16 shots located at the locations of the thick dots in Figure $9 \mathrm{~d}$. The thin dots represent all the 357 shots available in one of the SEAM data subsets. The solid lines in Figures 9a $9 \mathrm{~b}$ depict the decimated receiver lines for each of the 3 shots shown. In all panels $9 \mathrm{a}-9 \mathrm{~d}$, the large dot indicates the surface projection of the CIP used for illustration, located at coordinates $\{x, y, z\}=\{23.450,11.425,2.38\} \mathrm{km}$. For this example we consider the azimuth reference vector oriented in the $x$ direction, i.e. $\mathbf{v}=\{1,0,0\}$.

Figure 7: A subset of the SEAM velocity model used for the imaging example in Figures $8,11 \mathrm{~d}$,

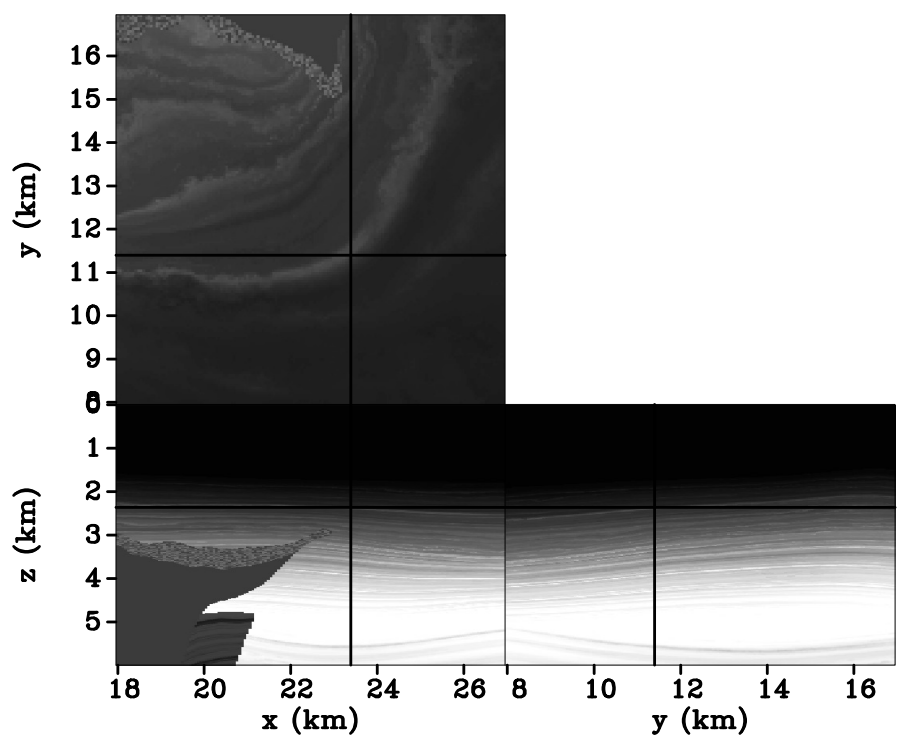

Figures 10a 10c show the extended image obtained at the CIP location indicated earlier using migration by downward continuation. The extended image cubes use 41 grid points in the $h_{x}$ and $h_{y}$ directions sampled on the image grid, i.e. at every $30 \mathrm{~m}$, and 31 grid points in the $\tau$ direction sampled on the data grid, i.e. at every $8 \mathrm{~ms}$. The vertical lag $h_{z}$ is not computed in this example, since the analyzed reflector is nearlyhorizontal. This lag is computed in the decomposition process from the horizontal lag and from the known information about the normal to the reflector at the given position. Figure 10d shows the extended image obtained for all 16 shots used for imaging. Although here we show the extended image cubes for independent shots, in practice these cubes need not be computed separately - the decomposition separates the information corresponds to different angles of incidence, as shown in this simple example.

Finally, Figures 11a 11d show the angle-domain decomposition of the extended image cubes shown in Figures $10 \mathrm{a}-10 \mathrm{~d}$, respectively. In these plots, the circles indi- 
Figure 8: Conventional image obtained using wavefield extrapolation with the 16 shots shown in Figure $9 \mathrm{~d}$.

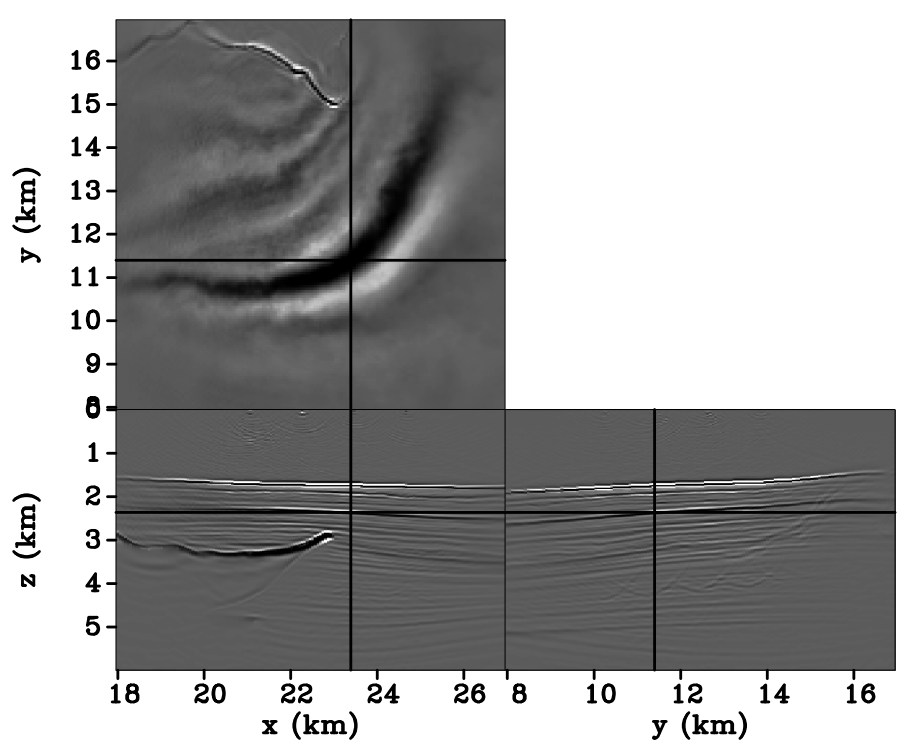

cating the reflection angles are drawn at every $5^{\circ}$ and the radial lines indicating the azimuth directions are drawn at every $15^{\circ}$. Given the sparse shot sampling, the CIP is sparsely illuminated, but at the correct reflection and azimuth angles.

\section{DISCUSSION}

We do not suggest in this paper that the wavefields used for imaging are planar prior to the interaction with the reflector. In complex geology, such an assumption would be unrealistic. However, a wavefield of arbitrary shape can be thought of as a superposition of plane waves propagating in various directions, either because the wavefronts characterizing the wavefields have curvature, or because the wavefields have triplicated during propagation. Each incident plane has a corresponding reflected plane related through Snell's law. Some angle decomposition techniques make use explicitly of a planar decomposition of the wavefields, followed of selection through thresholding of the most energetic plane (Xu et al., 2010). In contrast, we rely on the fact that all planar components of the wavefields have been transformed as planar events in the extended images and rely on slant-stacks or equivalent methods to separate them as a function of azimuth and reflection angles.

As indicated in the preceding sections, we do not need to compute all space-lags at the considered CIP positions. We could compute just two of them, e.g. $\lambda_{x}$ and $\lambda_{y}$ as shown in the examples of this paper, and then reconstruct the third lag using the information given by the reflector normal at the CIP position, equation 18. If the reflector is nearly vertical, it may be more relevant to compute the vertical and one horizontal space-lags. Alternatively, we could avoid computing the reflector normal vector from the conventional image, but instead compute all three components of the space-lag vector $\boldsymbol{\lambda}$. In this case, as indicated by Sava and Vasconcelos (2011), we could 

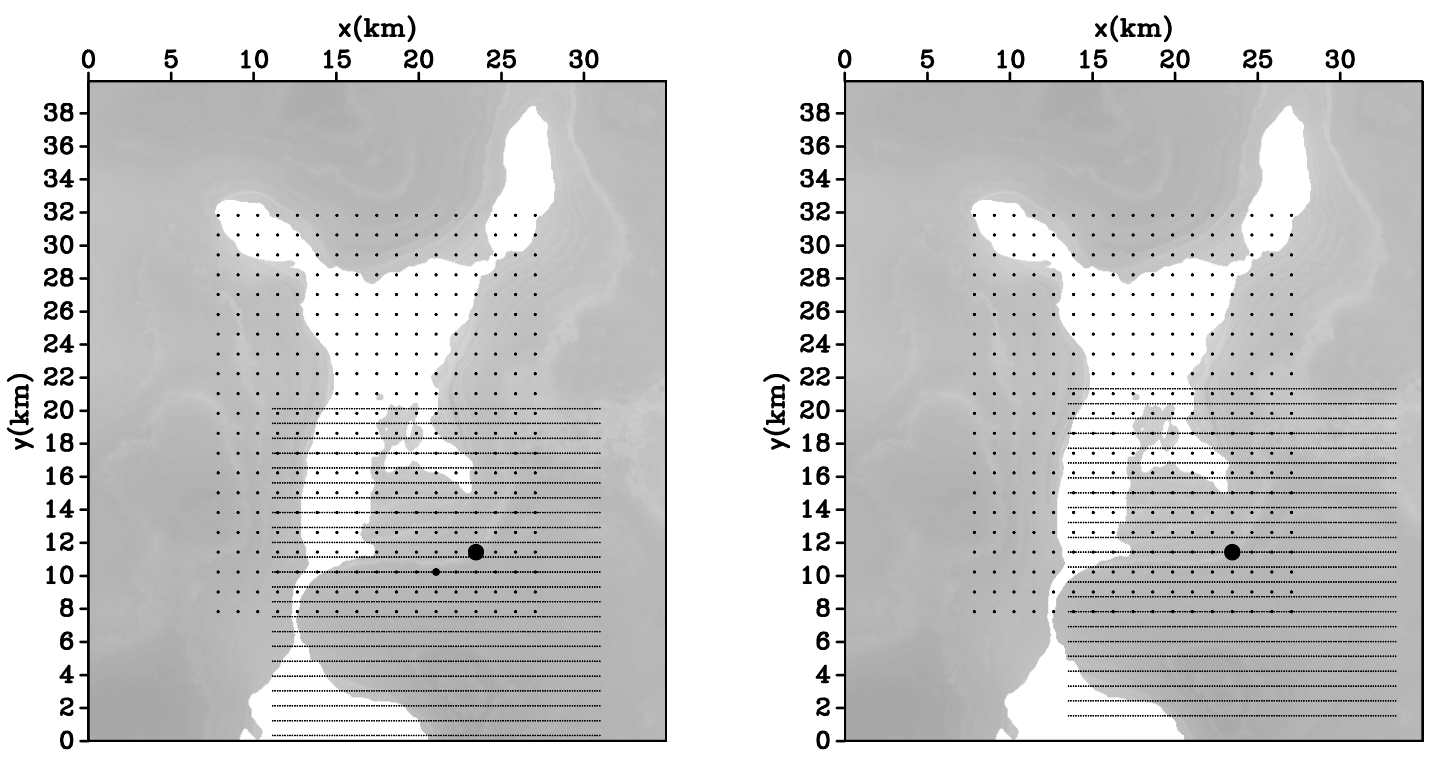

a

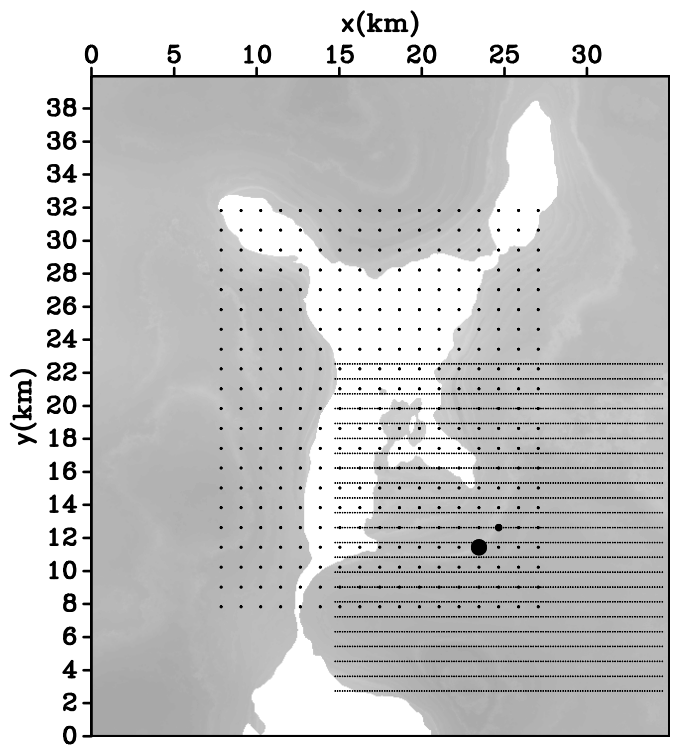

$\mathrm{C}$

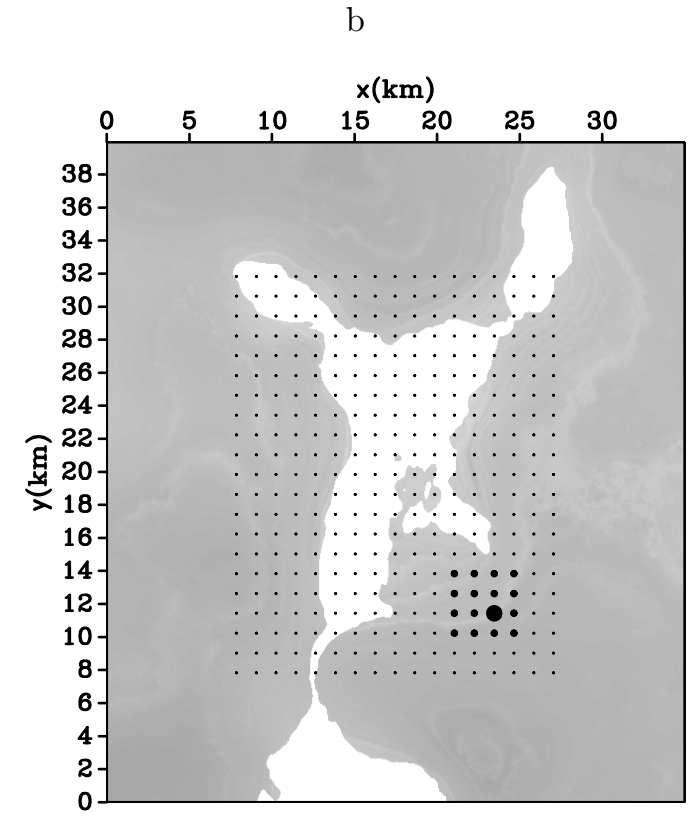

$\mathrm{d}$

Figure 9: Geometry of SEAM imaging experiment. Panels (a)-(c) show the position of one shot and the associated receiver lines (decimated by a factor of 30 in the $y$ direction. Panel $(d)$ shows the locations of the 16 shots used for creating the image shown in Figure 8. 


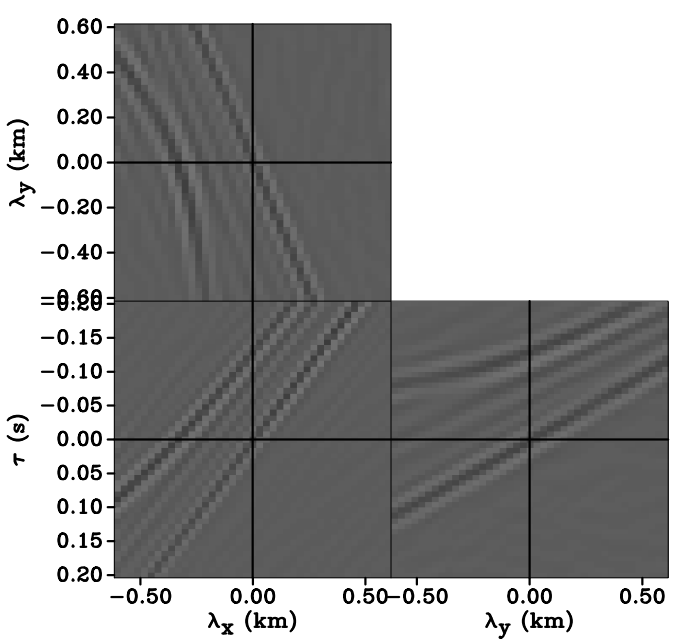

a

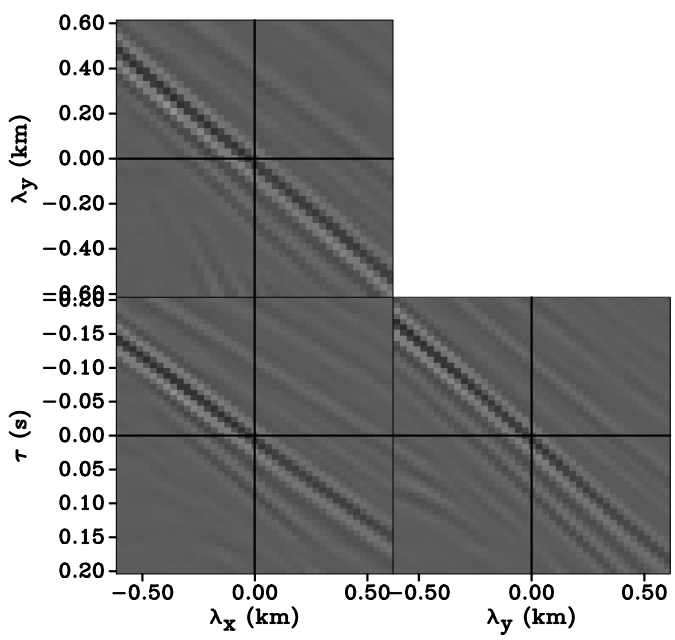

$\mathrm{c}$

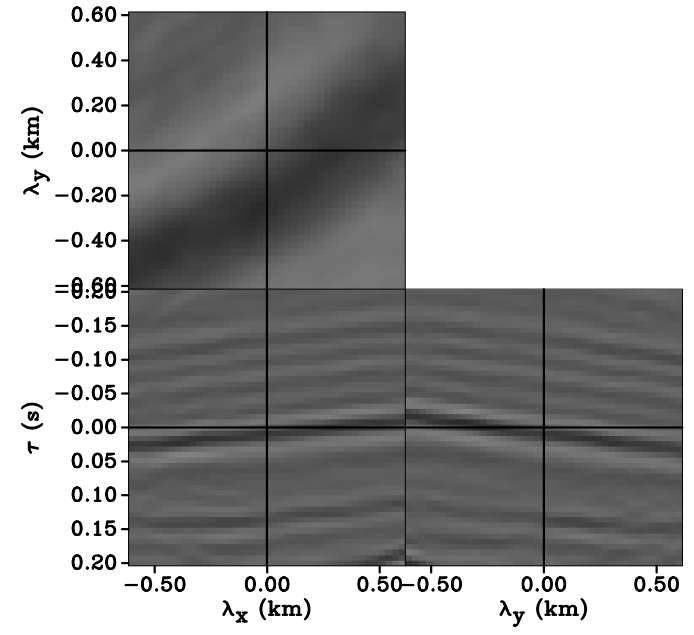

$\mathrm{b}$

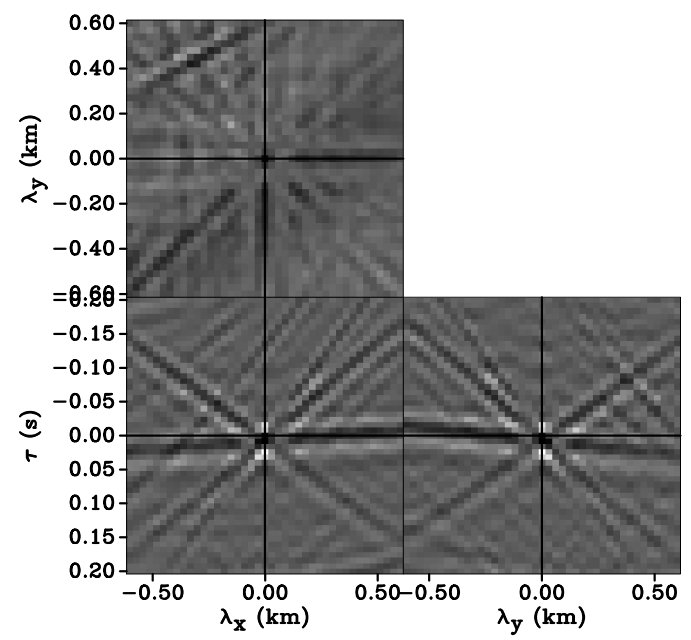

d

Figure 10: Extended image cubes for the SEAM imaging experiment. Panels (a)-(c) show extended image cubes at the same location for 3 different shots, and panel (d) shows the extended image obtained for all 16 shots considered in this experiment. 


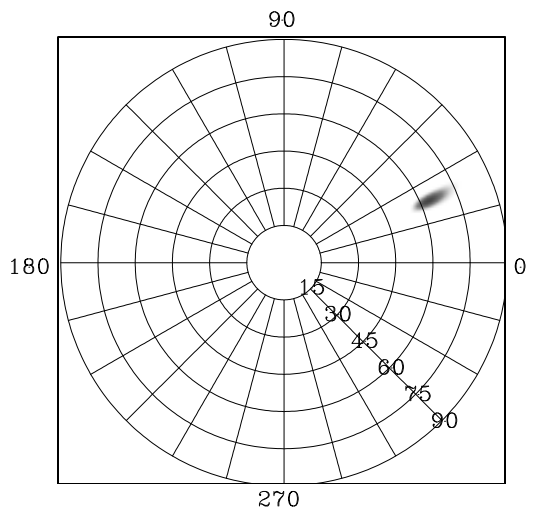

a

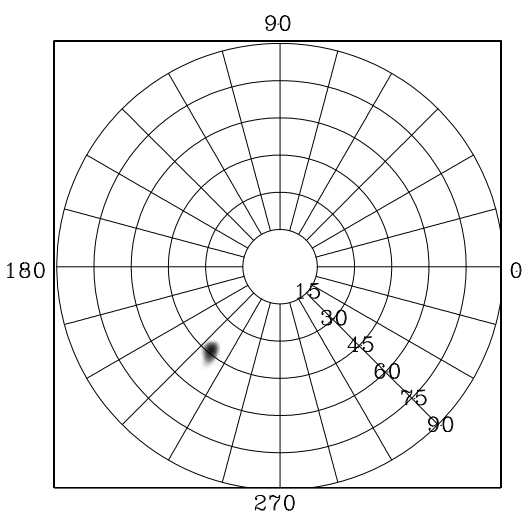

$\mathrm{c}$

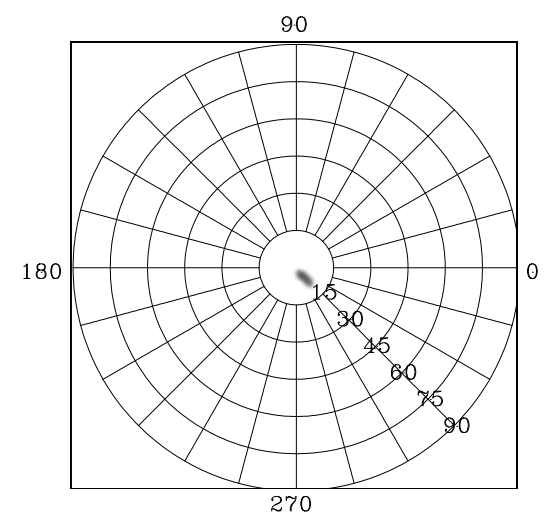

$\mathrm{b}$

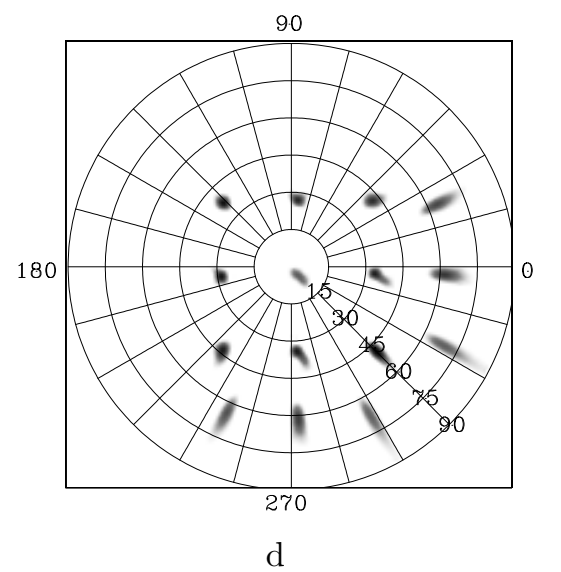

Figure 11: Reflectivity as a function of reflection and azimuth angles for the SEAM imaging experiment. Panels (a)-(c) show the angle-domain CIPs at the same location for 3 different shots, and panel (d) shows the angle-domain CIP obtained for all 16 shots considered in this experiment. The angles $\phi$ and $\theta$ are indexed along the contours using the trigonometric convention and along the radial lines increasing from the center. 
estimate the reflector dip from the lag information prior to the angle decomposition.

We have also noted earlier in the paper that the relevant space-lags are constructed in the reflector plane. This fact is a direct consequence of the fact that we have considered equal but with opposite sign time-shift of the source and receiver wavefields. Without this convention, the angle decomposition problem becomes more complex. In our experience to date, we did not find the need to relax this requirement.

The angle-domain CIPs accurately indicate the sampling of the reflector as a function of azimuth and reflection angles. If the shot distribution is sparse, or if the sub-surface geology creates shadow zones, the illumination is also sparse. This is both beneficial, assuming that the angle-domain CIPs are used to evaluate illumination, but it can also be a drawback if the angle-domain CIPs are used for AVA or MVA. However, a sparse sampling of a reflector is not a feature of the angle decomposition, but a feature of the acquisition geometry. Neither our, nor any other angle decomposition, can compensate for the lack of adequate data illuminating the subsurface on a dense angular grid.

Finally, we note that the most likely applications for angle decomposition in complex geology is the study of the reflector illumination itself. Assuming that the sampling is sufficiently dense and that the imaging velocity is accurately known, then we can use the angle decomposition discussed in this paper to evaluate amplitude variation with azimuth and reflection angles. However, we emphasize that this is a relevant exercise only if the reflector illumination is sufficiently dense. Otherwise, AVA effects overlap with illumination effects, rendering the analysis unreliable. Migration velocity analysis in the angle domain may also suffer from the lack of adequate illumination. This partial illumination may deteriorate the moveout which would otherwise be observed in the extended image domain. Furthermore, we do not advocate an implementation of MVA in the angle-domain, but rather in the extended image domain which contains all the relevant information and avoids the additional step of angle decomposition. An extensive discussion of this problem is outside the scope of our paper.

\section{CONCLUSIONS}

Angle decomposition based on wavefield extrapolation methods is characterized by robustness in areas with sharp velocity variation and by accuracy in the presence of steeply dipping reflectors. Extended common-image-point gathers constructed at discrete image points provide sufficient information for angle decomposition. The decomposition is based on the planar approximation of the source and receiver wavefields in the immediate vicinity of the image points. Both space-lag and the time-lag extensions are required to completely characterize the reflection geometry given by the local reflection and azimuth angles. However, assuming that information about the reflector slope is available, we could avoid computing one lag of the extended image, usually the vertical. This increases the computational efficiency of the method 
and makes it affordable for large-scale wide-azimuth imaging projects.

\section{ACKNOWLEDGMENTS}

Paul Sava acknowledges the support of the sponsors of the Center for Wave Phenomena at Colorado School of Mines. Ioan Vlad acknowledges Statoil for support of this research. The reproducible numeric examples in this paper use the Madagascar opensource software package freely available from http://www.reproducibility.org. The wide-azimuth data are property of SEG Advanced Modeling Corp (SEAM) and were provided to CWP by ExxonMobil Upstream Research.

\section{REFERENCES}

Berkhout, A. J., 1982, Imaging of acoustic energy by wave field extrapolation: Elsevier.

Biondi, B., and P. Sava, 1999, Wave-equation migration velocity analysis: 69th Annual International Meeting, SEG, Expanded Abstracts, 1723-1726.

Biondi, B., and W. Symes, 2004, Angle-domain common-image gathers for migration velocity analysis by wavefield-continuation imaging: Geophysics, 69, 1283-1298.

Biondi, B., and T. Tisserant, 2004, 3D angle-domain common-image gathers for migration velocity analysis: Geophys. Prosp., 52, 575-591.

Brandsberg-Dahl, S., M. V. de Hoop, and B. Ursin, 2003, Focusing in dip and AvA compensation on scattering-angle/azimuth common image gathers: Geophysics, 68, 232-254.

Clærbout, J. F., 1976, Fundamentals of geophysical data processing: Blackwell Scientific Publications.

- 1985, Imaging the Earth's interior: Blackwell Scientific Publications.

Clarke, R., G. Xia, N. Kabir, L. Sirgue, and S. Michell, 2006, Case study: A large 3D wide azimuth ocean bottom node survey in deepwater gom: SEG Technical Program Expanded Abstracts, 25, 1128-1132.

Clayton, R. W., and R. H. Stolt, 1981, A Born-WKBJ inversion method for acoustic reflection data: Geophysics, 46, 1559-1567.

de Bruin, C. G. M., C. P. A. Wapenaar, and A. J. Berkhout, 1990, Angle-dependent reflectivity by means of prestack migration: Geophysics, 55, 1223-1234.

Faye, J. P., and J. P. Jeannot, 1986, Prestack migration velocities from focusing depth analysis: 56th Ann. Internat. Mtg., Soc. of Expl. Geophys., Session:S7.6.

Gray, S. H., J. Etgen, J. Dellinger, and D. Whitmore, 2001, Seismic migration problems and solutions: Geophysics, 66, 1622-1640.

Koren, Z., I. Ravve, E. Ragoza, A. Bartana, P. Geophysical, and D. Kosloff, 2008, Full-azimuth angle domain imaging: SEG Technical Program Expanded Abstracts, 27, 2221-2225.

MacKay, S., and R. Abma, 1992, Imaging and velocity estimation with depth-focusing analysis: Geophysics, 57, 1608-1622. 
, 1993, Depth-focusing analysis using a wavefront-curvature criterion: Geophysics, 58, 1148-1156.

Michell, S., E. Shoshitaishvili, D. Chergotis, J. Sharp, and J. Etgen, 2006, Wide azimuth streamer imaging of mad dog; have we solved the subsalt imaging problem?: SEG Technical Program Expanded Abstracts, 25, 2905-2909.

Mosher, C. C., D. J. Foster, and S. Hassanzadeh, 1997, Common angle imaging with offset plane waves: 67th Ann. Internat. Mtg, Soc. of Expl. Geophys., 1379-1382.

Nemeth, T., 1995, Velocity estimation using tomographic depth-focusing analysis: 65th Ann. Internat. Mtg, Soc. of Expl. Geophys., 465-468.

—, 1996, Relating depth-focusing analysis to migration velocity analysis: 66th Ann. Internat. Mtg, Soc. of Expl. Geophys., 463-466.

Prucha, M., B. Biondi, and W. Symes, 1999, Angle-domain common image gathers by wave-equation migration: 69th Ann. Internat. Mtg, Soc. of Expl. Geophys., $824-827$.

Regone, C., 2006, Using 3D finite-difference modeling to design wide azimuth surveys for improved subsalt imaging: SEG Technical Program Expanded Abstracts, 25, 2896-2900.

Rickett, J., and P. Sava, 2002, Offset and angle-domain common image-point gathers for shot-profile migration: Geophysics, 67, 883-889.

Sava, P., and B. Biondi, 2004a, Wave-equation migration velocity analysis - I: Theory: Geophysical Prospecting, 52, 593-606.

— , 2004b, Wave-equation migration velocity analysis - II: Subsalt imaging examples: Geophysical Prospecting, 52, 607-623.

Sava, P., and S. Fomel, 2003, Angle-domain common image gathers by wavefield continuation methods: Geophysics, 68, 1065-1074.

—, 2005 , Coordinate-independent angle-gathers for wave equation migration: 75th Annual International Meeting, SEG, Expanded Abstracts, 2052-2055.

- 2006, Time-shift imaging condition in seismic migration: Geophysics, 71, S209-S217.

Sava, P., and I. Vasconcelos, 2011, Extended imaging condition for wave-equation migration: Geophysical Prospecting, 59, 35-55.

Shen, P., W. Symes, and C. C. Stolk, 2003, Differential semblance velocity analysis by wave-equation migration: 73rd Ann. Internat. Mtg., Soc. of Expl. Geophys., 2132-2135.

Shen, P., and W. W. Symes, 2008, Automatic velocity analysis via shot profile migration: Geophysics, 73, VE49-VE59.

Stolk, C. C., and W. W. Symes, 2004, Kinematic artifacts in prestack depth migration: Geophysics, 69, 562-575.

Stolt, R. H., and A. B. Weglein, 1985, Migration and inversion of seismic data: Geophysics, 50, 2458-2472.

Symes, W., 2009, Migration velocity analysis and waveform inversion: Geophysical Prospecting, 56, 765-790.

Weglein, A. B., and R. H. Stolt, 1999, Migration-inversion revisited (1999): The Leading Edge, 18, 950-952.

Wu, R.-S., and L. Chen, 2006, Directional illumination analysis using beamlet de- 
composition and propagation: Geophysics, 71, S147-S159.

Xie, X., and R. Wu, 2002, Extracting angle domain information from migrated wavefield: 72nd Ann. Internat. Mtg, Soc. of Expl. Geophys., 1360-1363.

Xu, S., H. Chauris, G. Lambare, and M. S. Noble, 1998, Common angle image gather: A new strategy for imaging complex media: 68th Ann. Internat. Mtg, Soc. of Expl. Geophys., 1538-1541.

Xu, S., Y. Zhang, and B. Tang, 2010, 3D common image gathers from reverse time migration: SEG Technical Program Expanded Abstracts, 29, 3257-3262.

Zhu, X., and R.-S. Wu, 2010, Imaging diffraction points using the local image matrices generated in prestack migration: Geophysics, 75, S1-S9.

\section{APPENDIX A}

This appendix shows the pseudo-code used for angle decomposition indicating the loop order and the link with the theory discussed in the body of the paper.

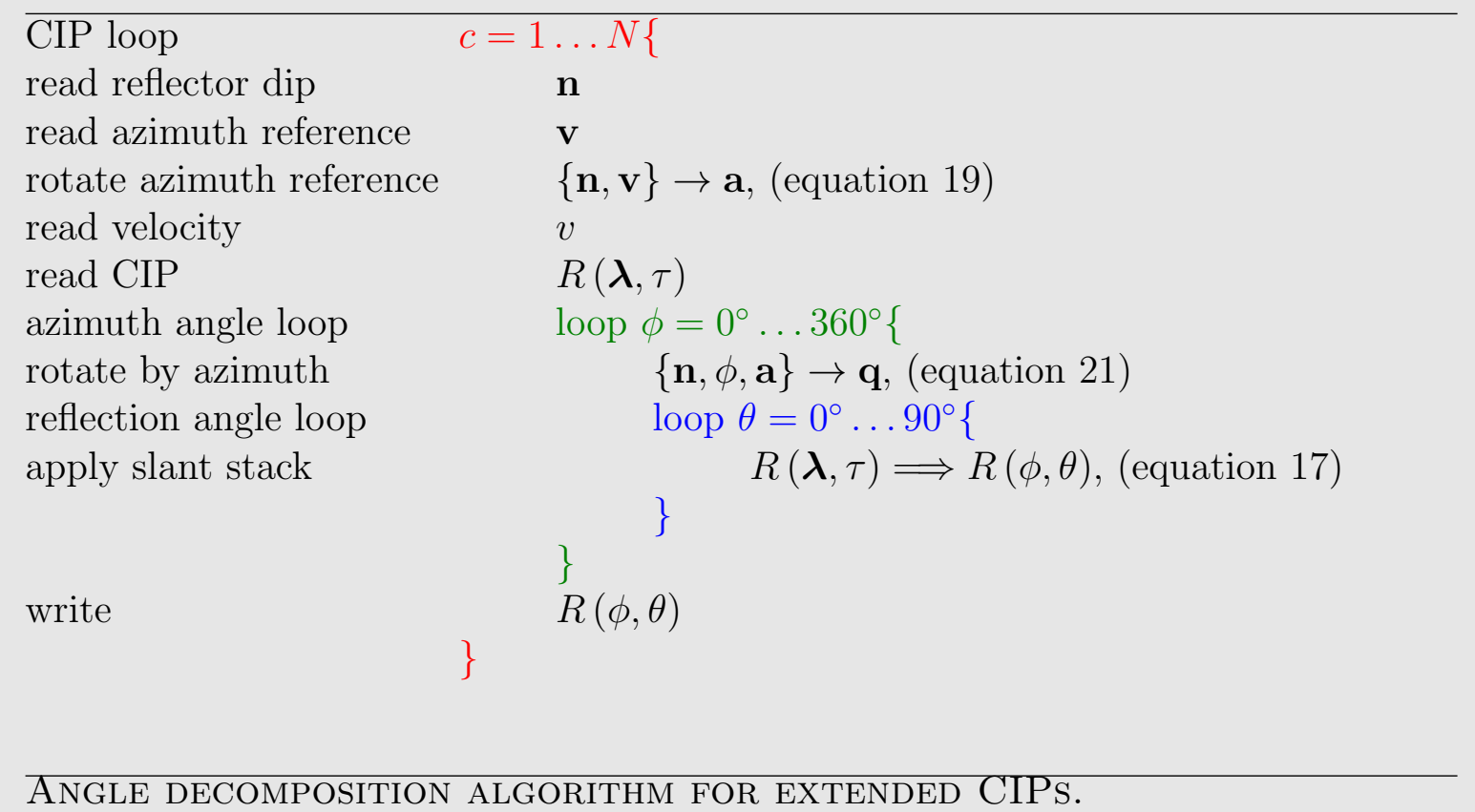

ANGLE DECOMPOSITION ALGORITHM FOR EXTENDED CIPS. 